\title{
Uncontrolled type 2 diabetes mellitus in Kandahar, Afghanistan: a cross-sectional analytical study
}

\section{ABSTRACT}

Background. Type 2 diabetes mellitus (T2DM) is one of the leading causes of mortality and morbidity worldwide. Main objective of this study was to determine the factors affecting uncontrolled T2DM.

Methods. This was a cross-sectional analytical study conducted in Kandahar, Afghanistan during July-December, 2018. Data was collected from 748 T2DM patients aged $>20$ years. Data was analyzed with SPSS software using descriptive statistics, Chi square test, and binary logistic regression.

Results. Among 748 T2DM patients, 390/748 (52.1\%) were females with 246/258 (95.3\%) having low or middle socio-economic status. Family history of DM was present in 402/746 (53.9\%) patients with 370/740 (50\%) patients having uncontrolled DM. Vanaspati or animal fat was used by $728 / 748(97.3 \%)$ of the patients, with $194 / 746$ (26\%) patients doing regular exercise. Oral hypoglycemic drugs were used by $666 / 720$ (92.5\%) of the patients. Comorbidities were present in $612 / 748$ $(81.8 \%)$ of the patients, with $348 / 748$ (46.5\%) having hypertension while $566 / 746$ (75.9\%) of the patients were either overweight or obese. Binary logistic regression revealed female gender (Adjusted Odds Ratio [AOR] 2.1, 95\% Cl 1.3-3.5), job without vigorous

Address for correspondence: Mohibullah Mako, MD, MPH Department of Public Health Faculty of Medicine, Kandahar University District 10, Durahi

Beside Aino Mena Town, Kandahar, Afghanistan

Phone: +93700351081

e-mail: mohib.mako31@gmail.com

Clinical Diabetology 2020, 9; 6: 416-425

DOI: $10.5603 /$ DK.2020.0053

Received: 19.06 .2020

Accepted: 02.11.2020 activity (AOR 2.2, 95\% Cl 1.3-3.6), and late diagnosis of DM (AOR 9.2, 95\% Cl 1.2-73.4) as the risk factors for uncontrolled T2DM.

Conclusion. Uncontrolled DM is prevalent in Kandahar. Proper control of the risk factors for uncontrolled DM will help in decreasing the severity and complications of DM. Diabetic services improvement, especially public awareness programs on media, is highly recommended to improve diabetic care in Kandahar. (Clin Diabetol 2020; 9; 6: 416-425)

Key words: diabetes mellitus, DM, Kandahar, Afghanistan, risk factors, determinants

\section{Introduction}

Type 2 diabetes mellitus (T2DM) is one of the leading cause of mortality and increases the risks of cardiovascular disease, blindness, kidney failure, and lower limb amputation [1]. In 2014, according to World Health Organization (WHO), approximately 422 million people worldwide had diabetes mellitus (DM) as compared to 108 million in 1980, particularly in low- and middle-income countries [1]. Approximately $90 \%$ of diabetes patients have T2DM, which is mostly related to lifestyle [2]. DM can cause many long-term complications in different parts of the body and can increase the overall risk of premature death [3]. Due to the increasing prevalence of obesity, especially among younger adults, T2DM is now more frequently diagnosed in young adults and adolescents, especially in high-income countries $[4,5]$. In 2010, prevalence of DM in Afghanistan among the age group of 20-79 years was estimated to be $8.6 \%$, whereas by 2030 it is estimated to reach $9.9 \%$ [6]. Moreover, studies reported that the prevalence of DM was $9.9 \%$ in Herat [7] 
$11.8 \%$ in Jalalabad [8], $13.2 \%$ in Kabul [9], and $22.4 \%$ in Kandahar [10]. Main objective of this study was to determine the factors affecting uncontrolled T2DM, as well as the sociodemographic, behavior, physical activity, and nutrition status of patients with T2DM in Kandahar, Afghanistan.

\section{Methods}

This was a cross-sectional analytical study. Data was collected during 6-month-period (July-December, 2018) using researcher-made questionnaire with questions regarding general characteristics, socio-economic status, physical activity, and nutrition of the diabetic patients.

The study population was composed of patients with T2DM with age $>20$ years who visited public and private health facilities in Kandahar, Afghanistan. All the patients were living in urban area (Kandahar city).

Research question: What are the factors affecting uncontrolled T2DM in Kandahar city, Afghanistan?

Primary objective was to determine the factors affecting uncontrolled T2DM in Kandahar city, Afghanistan.

Secondary objective was to determine the sociodemographic, behavior, physical activity, and nutrition status of patients with T2DM in Kandahar city. Inclusion criteria:

- patient with laboratory confirmed T2DM;

- both male and female patients with age $>20$ years;

- permanent residents of Kandahar city. Exclusion criteria:

- type 1 DM;

- patients who refused to consent for interview. Sample size was calculated using the following formula:

Where $\mathrm{n}$ is the sample size, $\mathrm{p}$ is the prevalence of outcome expressed as a proportion, $\mathrm{E}$ is the margin of error which is 0.05 in this case, 1.96 is the standard normal z-value corresponding to the $95 \%$ confidence interval.

The sample size and power calculations have been performed in Stata 15 (College Station, Texas, USA). Our sample size was 748 patients.

Written informed consents were taken from all the participants prior to the study. Information of the participants will not be disclosed. Ethical approval was taken from Kandahar University Ethics Committee with code number of KDRU-EC-2019.329.

Data was analyzed with SPSS version 22 (Chicago, IL, USA). Descriptive statistics, such as percentages and proportions, were used to describe the sociodemographic and other variables of the study participants. Chi square test (using crude odd ratio [COR]) was used to study the association of different factors in uncontrolled diabetic patients. All variables that showed statistically significant association were put in binary logistic regression (using adjusted odd ratio [AOR]) to determine the factors affecting uncontrolled T2DM. $P$ value of $<0.05$ was considered statistically significant.

\section{Definitions}

Diabetes: A patient with fasting blood glucose of $\geq 126 \mathrm{mg} / \mathrm{dL}$.

Fasting: Not having anything to eat or drink (except water) for at least 8 hours before the test.

Late diagnosis of T2DM: A diabetes patient with at least one diabetes related comorbidities or complications within 6 months before diagnosis.

Uncontrolled diabetes: Fasting blood glucose level of $\geq 126 \mathrm{mg} / \mathrm{dL}$ and random blood glucose of $\geq 200$ $\mathrm{mg} / \mathrm{dL}$ on previous three continuous occasions of the patient's visit to hospital.

\section{Results}

This was a cross-sectional study with data collected from 748 T2DM patients who visited the public and private health facilities of Kandahar city during a period of 6 months. Mean (SD) age of all patients, males, and females were 57.3 (12.6) years, 58.0 (13.3) years, and 56.6 (11.8) years, respectively (Table 1 ). Approximately half $(360 / 736$ [48.9\%]) of the patients were in the age group of 40-59 years. Females (390/748 [52.1\%]) were more than males $(358 / 748[47.9 \%])$, with almost all (386/390 [99.0\%]) of the female patients being housewives. Socio-economic status of most (246/258 [95.3\%]) of the patients was low or middle income. More than half (402/746 [53.9\%]) of the patients had a family history of DM (Table 2).

Uncontrolled DM was observed in 370/740 (50\%) of the patients. Overall, $94 / 746(12.6 \%)$ of the patients were smoking, with 74/358 (20.7\%) males and 20/388 (5.2\%) females. Mouth sniff (locally called Naswar) was being used by $266 / 746$ (35.7\%) of the patients, with $184 / 356(51.7 \%)$ in males. Fruits and vegetables were used daily by $62 / 620(10 \%)$ and $96 / 674(14.2 \%)$ of the patients. Nearly all (728/748 [97.3\%]) of the patients were using Vanaspati or animal fat, with $194 / 746$ (26\%) of the patients doing regular exercise. For treatment, 666/720 (92.5\%) of the patients were treated with oral hypoglycemic drugs, 34/720 (4.7\%) with insulin, while $20(2.8 \%)$ of the patients did not use any drugs for treatment. Comorbidities were present in $612 / 748(81.8 \%)$ of the patients. Hypertension was present in $348 / 748$ (46.5\%) of the patient, while $566 / 746(75.9 \%)$ of the patients were either overweight or obese (Table 3$)$. 


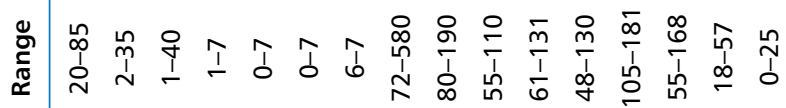

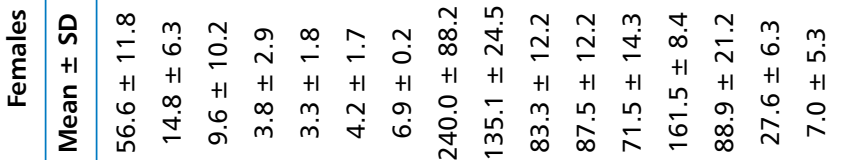

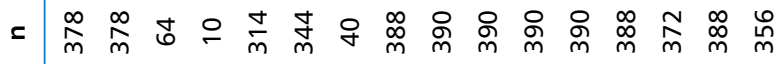

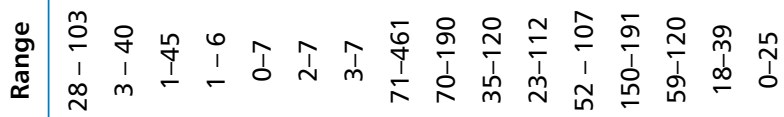

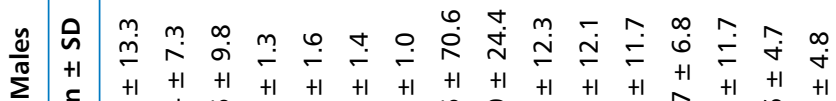

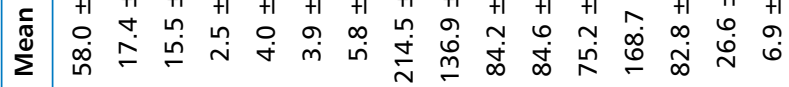

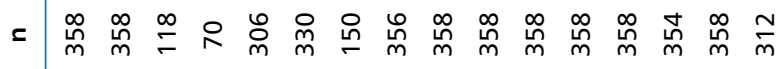

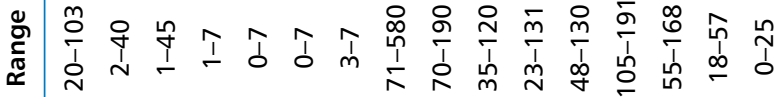

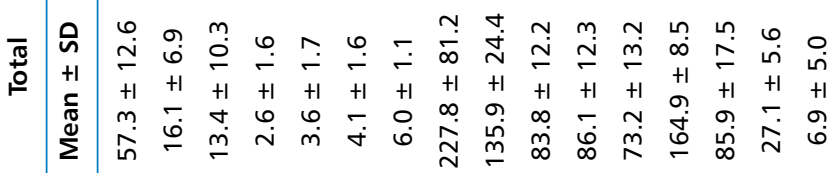

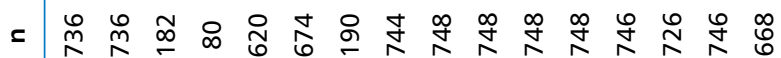

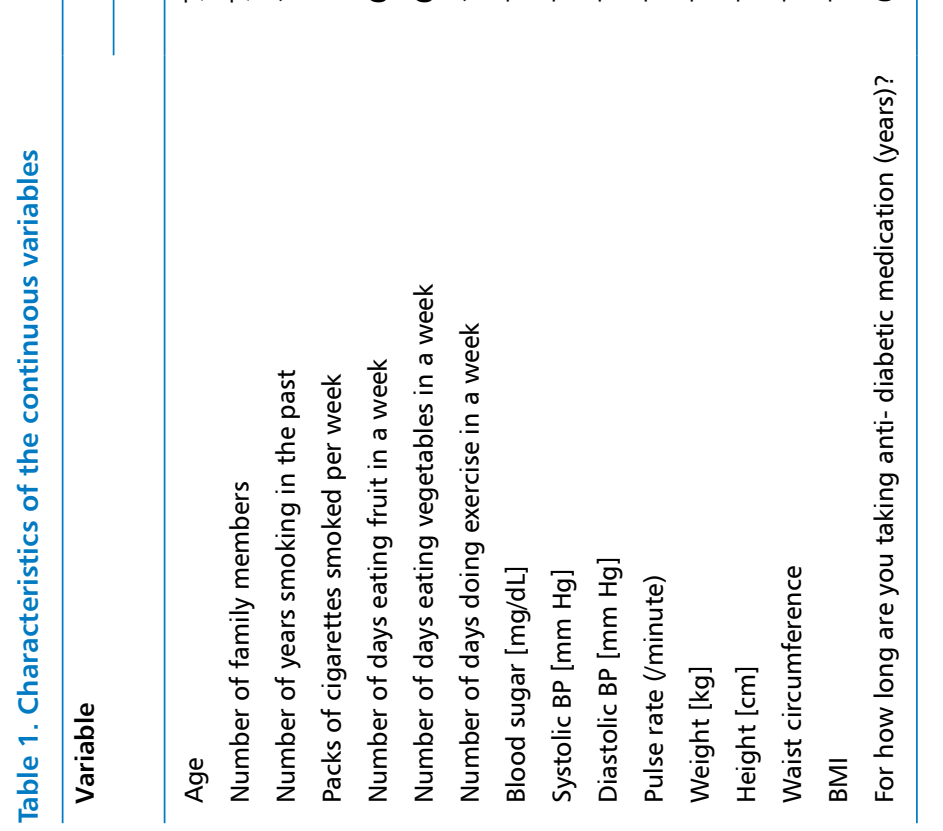


Table 2. Socio-demographic characteristics

\begin{tabular}{|c|c|c|c|}
\hline Variable & Total, n (\%) & Males, n (\%) & Females, n (\%) \\
\hline \multicolumn{4}{|l|}{ Age (years) } \\
\hline $20-39$ & $58(7.9)$ & $32(8.9)$ & $26(6.9)$ \\
\hline $40-59$ & $360(48.9)$ & $178(49.7)$ & $182(48.1)$ \\
\hline $60-79$ & $284(38.6)$ & $122(34.1)$ & $162(42.9)$ \\
\hline$>80$ & $34(4.6)$ & $26(7.3)$ & $8(2.1)$ \\
\hline Total & $736(100)$ & $358(100)$ & $378(100)$ \\
\hline \multicolumn{4}{|l|}{ Gender } \\
\hline Male & $358(47.9)$ & $358(100)$ & $0(0)$ \\
\hline Female & $390(52.1)$ & $0(0)$ & $390(100)$ \\
\hline Total & $748(100)$ & $358(100)$ & $390(100)$ \\
\hline \multicolumn{4}{|l|}{ Literacy } \\
\hline Literate & $362(48.4)$ & $280(78.2)$ & $82(21.0)$ \\
\hline Illiterate & $386(51.6)$ & $78(21.8)$ & $308(78.0)$ \\
\hline Total & $748(100)$ & $358(100)$ & $390(100)$ \\
\hline \multicolumn{4}{|l|}{ Marital status } \\
\hline Currently married & $486(65.0)$ & $278(77.6)$ & $208(53.3)$ \\
\hline Widowed & $242(32.4)$ & 70 (19.6) & $172(44.1)$ \\
\hline Never married & $16(2.1)$ & $10(2.8)$ & $6(1.6)$ \\
\hline Divorced & $4(0.5)$ & $0(0)$ & $4(1.0)$ \\
\hline Total & $748(100)$ & $358(100)$ & $390(100)$ \\
\hline \multicolumn{4}{|l|}{ Occupation } \\
\hline Government employee & $16(2.1)$ & $16(4.5)$ & $0(0.0)$ \\
\hline Non-government employee & $62(8.3)$ & $60(16.8)$ & $2(0.5)$ \\
\hline Self-employed & $160(21.4)$ & $158(44.1)$ & $2(0.5)$ \\
\hline Unemployed/Housewife & $510(68.2)$ & $124(34.6)$ & $386(99.0)$ \\
\hline Total & $748(100)$ & $358(100)$ & $390(100)$ \\
\hline \multicolumn{4}{|l|}{ Number of family members } \\
\hline$<5$ & $28(3.8)$ & $12(3.3)$ & $16(4.2)$ \\
\hline $5-9$ & $110(14.9)$ & $48(13.4)$ & $62(16.4)$ \\
\hline $10-19$ & $408(55.4)$ & $176(49.2)$ & $232(61.4)$ \\
\hline $20-30$ & $160(21.7)$ & $102(28.5)$ & $58(15.3)$ \\
\hline$>30$ & $30(4.1)$ & $20(5.6)$ & $10(2.7)$ \\
\hline Total & $637(100)$ & $358(100)$ & $378(100)$ \\
\hline \multicolumn{4}{|l|}{ Socio-economic status } \\
\hline Low income & $148(57.4)$ & $144(62.1)$ & $4(15.4)$ \\
\hline Middle income & $98(37.9)$ & $76(32.7)$ & $22(84.6)$ \\
\hline High income & $12(4.7)$ & $12(5.2)$ & $0(0.0)$ \\
\hline Total & $258(100)$ & $232(100)$ & $26(100)$ \\
\hline \multicolumn{4}{|l|}{ Family history of DM } \\
\hline Yes & $402(53.9)$ & $172(48.3)$ & $230(59.0)$ \\
\hline No & $344(46.1)$ & $184(51.7)$ & $160(41.0)$ \\
\hline Total & $746(100)$ & $356(100)$ & $390(100)$ \\
\hline \multicolumn{4}{|l|}{ Relative with history of DM } \\
\hline Brother & $124(30.8)$ & $62(35.6)$ & $62(27.2)$ \\
\hline Mother & $108(26.9)$ & $34(19.5)$ & $74(32.5)$ \\
\hline Father & $80(19.9)$ & $54(31.0)$ & $26(11.4)$ \\
\hline Sister & $60(14.9)$ & $12(6.9)$ & $48(21.0)$ \\
\hline Son & $14(3.5)$ & $8(4.6)$ & $6(2.6)$ \\
\hline Others* & $16(4.0)$ & $4(2.4)$ & $12(5.3)$ \\
\hline Total & $402(100)$ & $174(100)$ & $228(100)$ \\
\hline
\end{tabular}

*Other relatives: uncle, cousin, daughter, grandfather 
Table 3. Behavioral characteristics, DM-related characteristics, and physical measurements

\begin{tabular}{|c|c|c|c|}
\hline Variable & Total, n (\%) & Males, n (\%) & Females, n (\%) \\
\hline \multicolumn{4}{|l|}{ Current smoker } \\
\hline Yes & $94(12.6)$ & $74(20.7)$ & $20(5.2)$ \\
\hline No & $652(87.4)$ & $284(79.3)$ & $368(94.8)$ \\
\hline Total & $746(100)$ & $358(100)$ & $388(100)$ \\
\hline \multicolumn{4}{|c|}{ Has the doctor advised to stop smoking during last 12 months? } \\
\hline Yes & $84(91.3)$ & $70(97.2)$ & $14(70)$ \\
\hline No & $8(8.7)$ & $2(2.8)$ & $6(30)$ \\
\hline Total & $92(100)$ & $72(100)$ & $20(100)$ \\
\hline \multicolumn{4}{|c|}{ Currently using mouth sniff } \\
\hline Yes & $266(35.7)$ & $184(51.7)$ & $82(21.0)$ \\
\hline No & $480(64.3)$ & $172(48.3)$ & $308(78.0)$ \\
\hline Total & $746(100)$ & $356(100)$ & $390(100)$ \\
\hline \multicolumn{4}{|l|}{ Ex-smoker } \\
\hline Yes & $192(25.7)$ & $126(35.4)$ & $66(16.9)$ \\
\hline No & $554(74.3)$ & $230(64.6)$ & $324(83.1)$ \\
\hline Total & $746(100)$ & $356(100)$ & $390(100)$ \\
\hline \multicolumn{4}{|l|}{ Eating fruit } \\
\hline Every day in a week & $62(10.0)$ & $26(8.5)$ & $36(11.5)$ \\
\hline 3-6 days in a week & $436(70.3)$ & $234(76.5)$ & $202(64.3)$ \\
\hline$<3$ days in a week & $122(19.7)$ & $46(15.0)$ & $76(24.2)$ \\
\hline Total & $620(100)$ & $306(100)$ & $314(100)$ \\
\hline \multicolumn{4}{|l|}{ Eating vegetables } \\
\hline Every day & $96(14.2)$ & $32(9.7)$ & $64(18.6)$ \\
\hline 3-6 days in a week & $508(75.4)$ & $280(84.8)$ & $228(66.3)$ \\
\hline$<3$ days in a week & $70(10.4)$ & $18(5.5)$ & $52(15.1)$ \\
\hline Total & $674(100)$ & $330(100)$ & $344(100)$ \\
\hline \multicolumn{4}{|c|}{ Type of oil used for cooking } \\
\hline Vanaspati/animal fat & $728(97.3)$ & $342(95.5)$ & $386(99.0)$ \\
\hline Vegetable oil & $20(2.7)$ & $16(4.5)$ & $4(1.0)$ \\
\hline Total & $748(100)$ & $358(100)$ & $390(100)$ \\
\hline \multicolumn{4}{|l|}{ Job with vigorous activity } \\
\hline Yes & $270(36.1)$ & $192(53.6)$ & $78(20.0)$ \\
\hline No & $478(63.9)$ & $166(46.4)$ & $312(80.0)$ \\
\hline Total & $748(100)$ & $358(100)$ & $390(100)$ \\
\hline \multicolumn{4}{|l|}{ Exercise regularly } \\
\hline Yes & $194(26.0)$ & $156(43.8)$ & $38(9.7)$ \\
\hline No & $552(74.0)$ & $200(56.2)$ & $352(90.3)$ \\
\hline Total & $746(100)$ & $356(100)$ & $390(100)$ \\
\hline \multicolumn{4}{|l|}{ Type of exercise } \\
\hline Walking & $178(23.9)$ & $138(38.8)$ & $38(9.7)$ \\
\hline Running & $10(1.3)$ & $12(3.4)$ & $0(0.0)$ \\
\hline Body building & $6(0.8)$ & $6(1.7)$ & $0(0.0)$ \\
\hline No exercise & $552(74.0)$ & $200(56.2)$ & $352(90.3)$ \\
\hline Total & $746(100)$ & $356(100)$ & $390(100)$ \\
\hline \multicolumn{4}{|l|}{ Late diagnosis of DM } \\
\hline Yes & $732(98.1)$ & $346(97.2)$ & $386(99.0)$ \\
\hline No & $14(1.9)$ & $10(2.8)$ & $4(1.0)$ \\
\hline Total & $746(100)$ & $356(100)$ & $390(100)$ \\
\hline \multicolumn{4}{|c|}{ Taking antidiabetic medicine } \\
\hline Yes & $700(95.6)$ & $332(99.0)$ & $368(95.3)$ \\
\hline No & $32(4.4)$ & $14(4.0)$ & $18(4.7)$ \\
\hline Total & $732(100)$ & $346(100)$ & $386(100)$ \\
\hline
\end{tabular}


Table 3 (cont.). Behavioral characteristics, DM-related characteristics, and physical measurements

\begin{tabular}{|c|c|c|c|}
\hline Variable & Total, n (\%) & Males, n (\%) & Females, n (\%) \\
\hline Yes & 700 (95.6) & $332(99.0)$ & $368(95.3)$ \\
\hline No & $32(4.4)$ & $14(4.0)$ & $18(4.7)$ \\
\hline Total & $732(100)$ & $346(100)$ & $386(100)$ \\
\hline \multicolumn{4}{|l|}{ Type of DM medication } \\
\hline Oral hypoglycemic & $666(92.5)$ & $314(92.4)$ & $352(92.6)$ \\
\hline Insulin & $34(4.7)$ & $18(5.3)$ & $16(4.2)$ \\
\hline Non & $20(2.8)$ & $8(2.3)$ & $12(3.2)$ \\
\hline Total & $720(100)$ & $340(100)$ & $380(100)$ \\
\hline \multicolumn{4}{|l|}{ DM now under control } \\
\hline Yes & $370(50)$ & $208(59.1)$ & $162(41.8)$ \\
\hline No & $370(50)$ & $144(40.9)$ & $226(58.2)$ \\
\hline Total & $740(100)$ & $352(100)$ & $388(100)$ \\
\hline \multicolumn{4}{|l|}{ Co-morbidities present } \\
\hline Yes & $612(81.8)$ & $272(76.0)$ & $340(87.2)$ \\
\hline No & $136(18.2)$ & $86(24.0)$ & $50(12.8)$ \\
\hline Total & $748(100)$ & $358(100)$ & $390(100)$ \\
\hline \multicolumn{4}{|l|}{ Co-morbid diseases } \\
\hline HTN & $329(53.8)$ & $109(40.0)$ & $220(64.7)$ \\
\hline MI & $68(11.1)$ & $10(3.6)$ & $58(17.1)$ \\
\hline COPD & $60(9.8)$ & $49(18.2)$ & $11(3.2)$ \\
\hline Anxiety & $47(7.7)$ & $35(12.9)$ & $12(3.5)$ \\
\hline IHD & $47(7.7)$ & $30(11.0)$ & $17(5.0)$ \\
\hline Others* & $61(9.9)$ & $39(14.3)$ & $22(6.5)$ \\
\hline Total & $612(100)$ & $272(100)$ & $340(100)$ \\
\hline \multicolumn{4}{|l|}{ Blood pressure } \\
\hline Normal & $400(53.5)$ & $198(55.3)$ & $202(51.8)$ \\
\hline Stage 1 (mild) hypertension & $154(20.6)$ & $70(19.6)$ & $84(21.5)$ \\
\hline Stage 2 (moderate) hypertension & $144(19.2)$ & $58(16.2)$ & $86(22.1)$ \\
\hline Stage 3 (severe) hypertension & $50(6.7)$ & $32(8.9)$ & $18(4.6)$ \\
\hline Total & $748(100)$ & $358(100)$ & $390(100)$ \\
\hline \multicolumn{4}{|l|}{ BMI } \\
\hline Normal & $180(24.1)$ & $94(26.2)$ & $86(22.2)$ \\
\hline Overweight & $160(21.4)$ & $74(20.7)$ & $86(22.2)$ \\
\hline Obese & $406(54.5)$ & $190(53.1)$ & $216(55.6)$ \\
\hline Total & 746 (100) & $358(100)$ & $388(100)$ \\
\hline
\end{tabular}

DM — diabetes mellitus; COPD — chronic obstructive pulmonary disease; HTN — hypertension; IHD — ischemic heart disease; MI — myocardial infarction *Other comorbid diseases: stroke, chronic kidney injury, dyslipidemia

Chi-square test of the variables was done to determine the factors associated with uncontrolled T2DM. Statistically significant factors associated with uncontrolled T2DM were age $\geq 60$ years (COR 1.6, 95\% Cl 1.2-2.1; $P=0.002$ ), female gender (COR 2.0, 95\% Cl 1.5-2.7; $\mathrm{P}<0.001)$, unemployed/housewife (COR 2.0, 95\% $\mathrm{Cl} 1.4-2.7 ; \mathrm{P}<0.001)$, eating fruit $<3$ days in a week (COR 1.7, 95\% Cl 1.2-2.6; $\mathrm{P}=0.006$ ), job without vigorous activity (COR $2.7,95 \% \mathrm{Cl} 2.0-3.7$; $P<0.001)$, not doing regular exercise (COR 1.7, 95\% $\mathrm{Cl} 1.2-2.4 ; \mathrm{P}=0.001)$, late diagnosis of DM (COR 9.2,
$95 \% \mathrm{Cl} 1.2-73.4 ; \mathrm{P}=0.011)$, not taking antidiabetic drugs (COR 2.3, 95\% Cl 1.1-5.0; $\mathrm{P}=0.025)$, taking insulin (COR 2.0, 95\% Cl 1.0-4.1; P = 0.049), and DM that has affected routine of the patient (COR 1.6, 95\% Cl 1.1-2.3; P = 0.016) (Table 4).

Binary logistic regression of the above-mentioned statistically significant variables revealed female gender (AOR 2.1, 95\% Cl 1.3-3.5; $\mathrm{P}=0.004$ ), job without vigorous activity ( $A O R 2.2,95 \% \mathrm{Cl} 1.3-3.6 ; \mathrm{P}=0.003$ ), and late diagnosis of DM (AOR 9.2, 95\% Cl 1.2-73.4; $\mathrm{P}=0.035$ ) as the risk factors for uncontrolled T2DM (Table 5). 
Table 4. Chi-square test of the factors affecting uncontrolled T2DM

\begin{tabular}{|c|c|c|c|c|c|c|}
\hline Variable & Total, n (\%) & $\begin{array}{l}\text { Controlled } \\
\text { T2DM, n (\%) }\end{array}$ & $\begin{array}{l}\text { Uncontrolled } \\
\text { T2DM, n (\%) }\end{array}$ & COR & $95 \% \mathrm{Cl}$ & P value \\
\hline \multicolumn{7}{|l|}{ Age (years) } \\
\hline$<60$ & $418(55.9)$ & $224(62.2)$ & $188(51.1)$ & & & \\
\hline$\geq 60$ & $318(42.5)$ & $136(37.8)$ & $180(48.9)$ & 1.6 & $1.2-2.1$ & 0.002 \\
\hline Total & $736(100)$ & $360(100)$ & $368(100)$ & & & \\
\hline \multicolumn{7}{|l|}{ Gender } \\
\hline Male & $358(47.9)$ & $208(56.2)$ & $144(38.9)$ & & & \\
\hline Female & $390(52.1)$ & $162(43.8)$ & $226(61.1)$ & 2.0 & $1.5-2.7$ & $<0.001$ \\
\hline Total & $748(100)$ & $370(100)$ & $370(100)$ & & & \\
\hline \multicolumn{7}{|l|}{ Literacy } \\
\hline Literate & $362(48.4)$ & $170(45.9)$ & $212(57.3)$ & & & \\
\hline Illiterate & $386(51.6)$ & $200(54.1)$ & $158(42.7)$ & 0.6 & $0.5-0.8$ & 0.002 \\
\hline Total & $748(100)$ & $370(100)$ & $370(100)$ & & & \\
\hline \multicolumn{7}{|l|}{ Marital status } \\
\hline Single & $262(35.0)$ & $112(30.3)$ & $150(40.5)$ & & & \\
\hline Married & $486(65.0)$ & $258(69.7)$ & $220(59.5)$ & 0.6 & $0.5-0.9$ & 0.003 \\
\hline Total & $748(100)$ & $370(100)$ & $370(100)$ & & & \\
\hline \multicolumn{7}{|l|}{ Occupation } \\
\hline Employed & $238(31.8)$ & $144(38.9)$ & $90(24.3)$ & & & \\
\hline Unemployed/Housewife & $510(68.2)$ & $226(61.1)$ & $280(75.7)$ & 2.0 & $1.4-2.7$ & $<0.001$ \\
\hline Total & $748(100)$ & $370(100)$ & $370(100)$ & & & \\
\hline \multicolumn{7}{|l|}{ Number of family members } \\
\hline$<5$ & $28(3.8)$ & $8(2.2)$ & $20(5.6)$ & & & \\
\hline$\geq 5$ & $708(96.2)$ & $360(97.8)$ & $340(94.4)$ & 0.4 & $0.2-0.9$ & 0.018 \\
\hline Total & $637(100)$ & $368(100)$ & $360(100)$ & & & \\
\hline \multicolumn{7}{|l|}{ Socio-economic status } \\
\hline Low/Middle income & $246(95.3)$ & $154(96.3)$ & $90(93.8)$ & & & \\
\hline High income & $12(4.7)$ & $6(3.7)$ & $6(6.2)$ & 1.7 & $0.5-5.5$ & 0.360 \\
\hline Total & $258(100)$ & $160(100)$ & $96(100)$ & & & \\
\hline \multicolumn{7}{|l|}{ Family history of DM } \\
\hline Yes & $402(53.9)$ & $178(48.1)$ & $222(60.0)$ & & & \\
\hline No & $344(46.1)$ & $192(51.9)$ & $148(40.0)$ & 0.6 & $0.5-0.8$ & 0.001 \\
\hline Total & $746(100)$ & $370(100)$ & $370(100)$ & & & \\
\hline \multicolumn{7}{|l|}{ Current smoker } \\
\hline Yes & $94(12.6)$ & $44(12.0)$ & $48(13.0)$ & & & \\
\hline No & $652(87.4)$ & $324(88.0)$ & $322(87.0)$ & 0.9 & $0.6-1.4$ & 0.676 \\
\hline Total & $746(100)$ & $368(100)$ & $370(100)$ & & & \\
\hline \multicolumn{7}{|c|}{$\begin{array}{l}\text { Has the doctor advised to stop smoking during last } \\
12 \text { months? }\end{array}$} \\
\hline Yes & $84(91.3)$ & $40(90.9)$ & $44(91.7)$ & & & \\
\hline No & $8(8.7)$ & $4(9.1)$ & $4(8.3)$ & 0.9 & $0.2-3.9$ & 0.898 \\
\hline Total & $92(100)$ & $44(100)$ & $48(100)$ & & & \\
\hline \multicolumn{7}{|l|}{ Currently using mouth sniff } \\
\hline Yes & $266(35.7)$ & $128(34.8)$ & $138(37.3)$ & & & \\
\hline No & $480(64.3)$ & $240(65.2)$ & $232(62.7)$ & 0.9 & $0.7-1.2$ & 0.477 \\
\hline Total & $746(100)$ & $368(100)$ & $370(100)$ & & & \\
\hline \multicolumn{7}{|l|}{ Eating fruit } \\
\hline 3-7 days in a week & $498(80.3)$ & $270(84.4)$ & $222(75.5)$ & & & \\
\hline$<3$ days in a week & $122(19.7)$ & $50(15.6)$ & $72(24.5)$ & 1.7 & $1.2-2.6$ & 0.006 \\
\hline Total & $620(100)$ & $320(100)$ & $294(100)$ & & & \\
\hline \multicolumn{7}{|l|}{ Eating vegetables } \\
\hline 3-7 days in a week & $604(89.6)$ & $314(89.7)$ & $284(89.9)$ & & & \\
\hline$<3$ days in a week & $70(10.4)$ & $36(10.3)$ & $32(10.1)$ & 1.0 & $0.6-1.6$ & 0.946 \\
\hline Total & $674(100)$ & $350(100)$ & $316(100)$ & & & \\
\hline
\end{tabular}


Table 4 (cont.). Chi-square test of the factors affecting uncontrolled T2DM

\begin{tabular}{|c|c|c|c|c|c|c|}
\hline Variable & Total, n (\%) & $\begin{array}{l}\text { Controlled } \\
\text { T2DM, n (\%) }\end{array}$ & $\begin{array}{l}\text { Uncontrolled } \\
\text { T2DM, n (\%) }\end{array}$ & COR & $95 \% \mathrm{Cl}$ & $P$ value \\
\hline \multicolumn{7}{|c|}{ Type of oil used for cooking } \\
\hline Vanaspati/animal fat & $728(97.3)$ & $364(98.4)$ & $356(96.2)$ & & & \\
\hline Vegetable oil & $20(2.7)$ & $6(1.6)$ & $14(3.8)$ & 2.4 & $0.9-6.3$ & 0.070 \\
\hline Total & $748(100)$ & $370(100)$ & $370(100)$ & & & \\
\hline \multicolumn{7}{|l|}{ Job with vigorous activity } \\
\hline Yes & $270(36.1)$ & $174(47.0)$ & $92(24.9)$ & & & \\
\hline No & $478(63.9)$ & $196(53.0)$ & $278(75.1)$ & 2.7 & $2.0-3.7$ & $<0.001$ \\
\hline Total & $748(100)$ & $370(100)$ & $370(100)$ & & & \\
\hline \multicolumn{7}{|l|}{ Exercise regularly } \\
\hline Yes & $194(26.0)$ & $114(31.0)$ & $76(20.5)$ & & & \\
\hline No & $552(74.0)$ & $254(69.0)$ & $294(79.5)$ & 1.7 & $1.2-2.4$ & 0.001 \\
\hline Total & $746(100)$ & $368(100)$ & $370(100)$ & & & \\
\hline \multicolumn{7}{|l|}{ Late diagnosis of DM } \\
\hline Yes & $732(98.1)$ & 369 (99.7) & 361 (97.6) & & & \\
\hline No & $14(1.9)$ & $1(0.3)$ & $9(2.4)$ & 9.2 & $1.2-73.4$ & 0.011 \\
\hline Total & $746(100)$ & $370(100)$ & $370(100)$ & & & \\
\hline \multicolumn{7}{|c|}{ Taking antidiabetic medicine } \\
\hline Yes & $700(95.6)$ & $360(97.3)$ & 338(93.9) & & & \\
\hline No & $32(4.4)$ & $10(2.7)$ & $22(6.1)$ & 2.3 & $1.1-5.0$ & 0.025 \\
\hline Total & $732(100)$ & $370(100)$ & $360(100)$ & & & \\
\hline \multicolumn{7}{|c|}{ Type of antidiabetic medicine } \\
\hline Oral antidiabetic & $666(95.1)$ & $348(96.7)$ & $316(93.5)$ & & & \\
\hline Insulin & $34(4.9)$ & $12(3.3)$ & $22(6.5)$ & 2.0 & $1.0-4.1$ & 0.051 \\
\hline Total & $700(100)$ & $360(100)$ & $338(100)$ & & & \\
\hline \multicolumn{7}{|l|}{ Co-morbidities present } \\
\hline Yes & $612(81.8)$ & $304(82.2)$ & $300(81.1)$ & & & \\
\hline No & $136(18.2)$ & $66(17.8)$ & $70(18.9)$ & 1.1 & $0.7-1.6$ & 0.704 \\
\hline Total & $748(100)$ & $370(100)$ & $370(100)$ & & & \\
\hline \multicolumn{7}{|l|}{ Blood pressure } \\
\hline Normal & $400(53.5)$ & $198(53.5)$ & $196(53.0)$ & & & \\
\hline Hypertension & $348(46.5)$ & $172(46.5)$ & $174(47.0)$ & 1.0 & $0.8-1.4$ & 0.883 \\
\hline Total & $748(100)$ & $370(100)$ & $370(100)$ & & & \\
\hline \multicolumn{7}{|l|}{ BMI } \\
\hline Normal & $180(24.1)$ & $80(21.6)$ & $98(26.6)$ & & & \\
\hline Overweight/Obese & $566(75.9)$ & $290(78.4)$ & $270(73.4)$ & 0.8 & $0.5-1.1$ & 0.112 \\
\hline Total & $746(100)$ & $370(100)$ & $368(100)$ & & & \\
\hline
\end{tabular}

$\mathrm{BMI}$ - body mass index; DM - diabetes mellitus; T2DM - type 2 diabetes mellitus

\section{Discussion}

In this cross-sectional study, we studied 748 T2DM patients to determine the factors in uncontrolled T2DM in Kandahar, Afghanistan. Although DM is prevalent in Afghanistan, until now very few studies have been conducted on this devastating disease [7-9]. To our knowledge, there has never been any study in Afghanistan to find out the factors affecting uncontrolled DM.

In our study, DM was uncontrolled in half (50\%) of the patients. This higher prevalence of uncon- trolled DM is of great concern, and is also broadly in line with studies from Ethiopia (50\%) [11] and Pakistan (38.9\%) [12]. Contrary, even higher prevalence of uncontrolled DM have been reported from Ghana (86.4\%) [3] and Saudi Arabia (74\%) [13]. This differences in prevalence of DM may be due to the differences in care, attitude, and practices among DM patients; different methods of health education, treatment, and counselling or variances in geographical regions [3]. 
Table 5. Binary logistic regression for estimating the factors affecting uncontrolled T2DM

\begin{tabular}{lccc}
\hline Variable & AOR & $95 \% \mathrm{Cl}$ & P value \\
\hline Job with vigorous activity & 2.2 & $1.3-3.6$ & 0.003 \\
Gender & 2.1 & $1.3-3.5$ & 0.004 \\
Late diagnosis of DM & 9.2 & $1.2-73.4$ & 0.035 \\
Taking antidiabetic medicine & 0.1 & $0.0-1.1$ & 0.055 \\
Type of antidiabetic medicine & 2.2 & $0.9-5.0$ & 0.067 \\
Age & 1.4 & $0.9-2.2$ & 0.106 \\
Eating fruit & 1.4 & $0.9-2.2$ & 0.173 \\
Occupation & 0.7 & $0.3-1.4$ & 0.279 \\
Exercise regularly & 0.9 & $0.5-1.5$ & 0.683 \\
\hline
\end{tabular}

DM - diabetes mellitus; T2DM - type 2 diabetes mellitus

In our study, uncontrolled DM was more prevalent (61.1\%) among females. Similar results have been reported from Jordan (51.9\%) [14], Ghana (76.8\%) [3] and Pakistan (77.3\%) [12].

Sedentary lifestyle and lack of regular exercise increases the risk of T2DM. In our study only $26 \%$ of the patients were doing regular exercise. Similarly, studies Saudi Arabia [15], Jordan [14], and USA [16] have also revealed that physical inactivity is the predictor of poor glycemic control. Physical exercise not only improves glycemic control, but also boosts patient's insulin sensitivity and repairs some of the damage due by DM associated complications, for instance impaired cardiovascular health [17].

Comorbidities were present in majority $(81.8 \%)$ of our patients. Similarly, most of the patients had comorbidities in Ghana (86,4\%) [3], Jordan (65.1\%) [14], and Saudi Arabia (65.0\%) [18]. In our study, main factors associated with uncontrolled T2DM were female gender, job without vigorous activity, and late diagnosis of DM. A study in Jordan revealed that statistically significant factors associated with uncontrolled DM were increased duration of DM, not following dietitians-recommended eating plan, negative attitude towards DM, and increased barriers to adherence scale scores [14]. A study conducted in Pakistan showed that patients aged $<50$ years, being diagnosed in a hospital rather than a clinic, diabetes information from a doctor or nurse only rather than multiple sources, higher monthly treatment cost, and higher consumption of tea as the main factors for uncontrolled DM. On the other hand, a study in Saudi Arabia concluded that a family history of DM, having longer diabetic durations, not doing sufficient physical exercise, and being overweight were the statistically significant risk factors [18]. These findings emphasize on the importance of patients at risk of developing complication due to DM and implementation of more effective preventive measure [19, 20].

Main limitations of our study were cross-sectional nature of the study (all risk factors of uncontrolled T2DM could not be studied, especially $\mathrm{HbA}_{1 \mathrm{c}}$ ), patients were mostly from urban area (we cannot generalize the results to the entire population), and inability to follow up the patients longitudinally.

\section{Conclusion}

Diabetes mellitus is prevalent in Kandahar, affecting females more than males. Half of the T2DM patients had uncontrolled DM. Main risk factors for uncontrolled DM were female gender, job without vigorous activity, and late diagnosis of DM. Proper control of these risk factors will help in decreasing the severity and complications of DM. Diabetic services improvement, especially public awareness programs on media, is highly recommended to improve diabetic care in Kandahar. Future studies (especially prospective studies) are needed in Kandahar to find out the different aspects of DM prevalence, clinical features, complications, diagnosis, management, and prognosis.

\section{Acknowledgements}

We present our highest and sincere thanks to the authorities of MPH program, Faculty of Medicine, Kandahar University, and Directorate of Public Health. We are also very thankful of the staff of health facilities in Kandahar and our study participants.

\section{Conflict of interests}

The authors report no conflicts of interest in this study.

\section{REFERENCES}

1. World Health Organization. Global Report on Diabetes. WHO. 2016. https://www.who.int/publications-detail/global-report-ondiabetes (20.02.2019).

2. Trapp CB, Barnard ND. Usefulness of vegetarian and vegan diets for treating type 2 diabetes. Curr Diab Rep. 2010; 10(2): 152-158, doi: 10.1007/s11892-010-0093-7, indexed in Pubmed: 20425575.

3. Fiagbe J, Takramah W, Axame W, et al. Risk factors associated with diabetes mellitus among adults in the Hohoe Municipality of Ghana. Journal of Advances in Medicine and Medical Research. 2017; 23(2): 1-12, doi: 10.9734/jammr/2017/33846.

4. Alberti G, Zimmet P, Shaw J, et al. Consensus Workshop Group. Type 2 diabetes in the young: the evolving epidemic: the international diabetes federation consensus workshop. Diabetes Care. 2004; 27(7): 1798-1811, doi: 10.2337/diacare.27.7.1798, indexed in Pubmed: 15220270.

5. Koopman RJ, Mainous AG, Diaz VA, et al. Changes in age at diagnosis of type 2 diabetes mellitus in the United States, 1988 to 2000. Ann Fam Med. 2005; 3(1): 60-63, doi: 10.1370/afm.214, indexed in Pubmed: 15671192.

6. Shaw JE, Sicree RA, Zimmet PZ. Global estimates of the prevalence of diabetes for 2010 and 2030. Diabetes Res Clin Pract. 2010; 87(1): 4-14, doi: 10.1016/j.diabres.2009.10.007, indexed in Pubmed: 19896746. 
7. Islam Saeed KM. Diabetes Mellitus Among Adults in Herat, Afghanistan: A Cross-Sectional Study. Cent Asian J Glob Health. 2017; 6(1): 271, doi: 10.5195/cajgh.2017.271, indexed in Pubmed: 29138737.

8. Khwaja S, Islam M. Prevalence and Predictors of Diabetes Mellitus in Jalalabad City, Afghanistan-2013. Iran J DIABETES Obes. 2014; 6 (1): 1-8.

9. Saeed K, Asghar R, Sahak M, et al. Prevalence and risk factors associated with diabetes mellitus among Kabul citizens - Afghanistan, 2012. International Journal of Diabetes in Developing Countries. 2015; 35(3): 297-303, doi: 10.1007/s13410-014-0270-3.

10. Mir K, Saeed I. Prevalence of Diabetes and its Risk Factors in Urban Setting of Kandahar City, Afghanistan-2015. IOSR J Pharm. 2016; 6(11): $53-60$

11. Woldu MA, Wami CD. Factors associated with poor glycemic control among patients with type 2 diabetes mellitus in Ambo Hospital, Ambo; Ethiopia. Endocrinology \& Metabolic Syndrome. 2014; 03(04), doi: 10.4172/2161-1017.1000143.

12. Siddiqui FJ, Avan BI, Mahmud S, et al. Uncontrolled diabetes mellitus: prevalence and risk factors among people with type 2 diabetes mellitus in an Urban District of Karachi, Pakistan. Diabetes Res Clin Pract. 2015; 107(1): 148-156, doi: 10.1016/j. diabres.2014.09.025, indexed in Pubmed: 25451895.

13. Almutairi MA, Said SM, Zainuddin H. Predictors of poor glycemic control among type two diabetic patients. Am J Medicine Medical Sci. 2013; 3(2): 17-21, doi: 10.5923/j.ajmms.20130302.01.

14. Khattab M, Khader YS, Al-Khawaldeh A, et al. Factors associated with poor glycemic control among patients with type 2 diabetes.
J Diabetes Complications. 2010; 24(2): 84-89, doi: 10.1016/j. jdiacomp.2008.12.008, indexed in Pubmed: 19282203.

15. Almutairi MA, Said SM, Zainuddin H. Predictors of Poor Glycemic Control Among Type Two Diabetic Patients. Am J Med Med Sci. 2013; 3(2): 17-21, doi: 10.5923/j.ajmms.20130302.01.

16. Daly JM, Hartz AJ, Xu Y, et al. An assessment of attitudes, behaviors, and outcomes of patients with type 2 diabetes. J Am Board Fam Med. 2009; 22(3): 280-290, doi: 10.3122/ jabfm.2009.03.080114, indexed in Pubmed: 19429734.

17. Thent ZC, Das S, Henry $\sqcup$. Role of exercise in the management of diabetes mellitus: the global scenario. PLoS One. 2013; 8(11): e80436, doi: 10.1371/journal.pone.0080436, indexed in Pubmed: 24236181.

18. Alzaheb RA, Altemani AH. The prevalence and determinants of poor glycemic control among adults with type 2 diabetes mellitus in Saudi Arabia. Diabetes Metab Syndr Obes. 2018; 11: 15-21, doi: 10.2147/ DMSO.S156214, indexed in Pubmed: 29430192.

19. Stratton IM, Adler Al, Neil HA, et al. Association of glycaemia with macrovascular and microvascular complications of type 2 diabetes (UKPDS 35): prospective observational study. BMJ. 2000; 321(7258): 405-412, doi: 10.1136/bmj.321.7258.405, indexed in Pubmed: 10938048.

20. Nathan DM, Bayless M, Cleary P, et al. DCCT/EDIC Research Group. Diabetes control and complications trial/epidemiology of diabetes interventions and complications study at 30 years: advances and contributions. Diabetes. 2013; 62(12): 3976-3986, doi: 10.2337/ db13-1093, indexed in Pubmed: 24264395. 